\title{
Sensitivity analysis of fluid substitution in a porous medium with aligned fractures
}

Samik Sil ${ }^{1}$, Mrinal K. Sen ${ }^{2}$, and Boris Gurevich ${ }^{3}$

1.ConocoPhillips, 2. University of Texas at Austin, 3. Curtin University

\section{Summary}

We study the effect of fluid substitution in a porous fractured medium using explicit expressions developed for aligned fractured medium. We investigate the effect of porosity and water saturation on (1) P-wave moduli, (2) horizontal and vertical velocities, (3) anisotropic parameters, and (4) reflection coefficients. Effects of fracture density on these four parameters are also analyzed. The systematic variations of the moduli and reflection coefficients reported in this paper can thus be used in developing AVO with azimuth in a porous fractured reservoir.

\section{Introduction}

Majority of land reservoirs are fractured to some extent. Conventional fractured reservoirs are porous; the porous matrix contains the hydrocarbon and embedded fractures act as permeable conduits (Nelson, 2001). Our paper focuses on these types of reservoirs. We assume that the embedded fracture system in the reservoir matrix is aligned along a preferred direction. Therefore the medium studied in this paper can be treated as porous and transversely isotropic with a horizontal axis of symmetry (HTI).

Fluid substitution in a porous HTI medium can be performed using Gassmann (1951) anisotropic fluid substitution equation in stiffness domain (considering low frequency limit). Brown and Korringa (1975) extended these equations to micro-inhomogeneous and microanisotropic media. Recently, Gurevich (2003) developed explicit analytical expressions for fluid substitution in a porous HTI medium. These equations are relatively easy to implement and free from any assumption on the extent of fracturing. Using Gurevich's (2003) HTI fluid substitution equations, we attempt to answer the following questions: how (1) P-wave moduli (anisotropic), (2) P-wave velocities (vertical and horizontal), (3) anisotropic parameter (Rüger, 1997), and (4) reflection coefficient are affected by changing background porosity and water saturation of the porous HTI medium. The effect of changing crack density on the above four parameters is also studied.

\section{Theory}

The theory of porous HTI medium fluid substitution is described in detail by Gurevich (2003). Here we present a brief review, for completeness. Along with the isotropic cases, Gassmann (1951) also presented an equation for fluid substitution in any anisotropic medium (applicable to the low frequency limit). The relationship between dry and saturated elastic moduli based on that anisotropic fluid substitution equation is:

$$
C_{i j}^{s a t}=C_{i j}^{d r y}+\alpha_{i} \alpha_{j} M . \quad i, j=1, \ldots .6 .
$$

In equation $1, \mathrm{C}_{\mathrm{ij}}{ }^{\mathrm{dry}}$ is the dry fame stiffness matrix of the anisotropic medium and $\alpha$ is a function of anisotropic Pwave modulus (Gurevich, 2003). The expression $M$ is a function of grain bulk modulus $K_{g}$, fluid bulk modulus $K_{f}$, porosity $\varphi$, and generalized drained bulk modulus $K^{*}$ of the anisotropic medium (Gurevich, 2003).

For the HTI medium, Gurevich (2003) further derived:

$$
\begin{aligned}
& \alpha_{1}=\alpha_{0}+\frac{K \Delta_{N}}{K_{g}}, \\
& \alpha_{2}=\alpha_{3}=\alpha_{0}+\frac{K \lambda \Delta_{N}}{K_{g}(\lambda+2 \mu)},
\end{aligned}
$$

and,

$$
K^{*}=K\left(1-\frac{K \Delta_{N}}{\lambda+2 \mu}\right) .
$$

Other parameters $\alpha_{4}=\alpha_{5}=\alpha_{6}=0$ for the HTI medium. In these equations $K=\lambda+2 \mu$ is the dry bulk modulus of the background isotropic rock, $\alpha_{0}=1-K / K g$, and $\Delta_{N}$ is the normal weakness (Schoenberg and Sayers, 1995) of the aligned vertical fracture sets. Now using all the above equations Gurevich (2003) obtained:

$$
\begin{aligned}
& C_{11}^{\text {Sat }}=\frac{L}{D}\left\{d_{1} \theta+\frac{K_{F}}{\varphi K_{g} L}\left[L_{1} \alpha^{\prime}-\frac{16 \mu^{2} \alpha_{0} \Delta_{N}}{9 L}\right]\right\}, \\
& C_{33}^{\text {Sat }}=\frac{L}{D}\left\{d_{2} \theta+\frac{K_{F}}{\varphi K_{g} L}\left[L_{1} \alpha^{\prime}-\frac{4 \mu^{2} \alpha_{0} \Delta_{N}}{9 L}\right]\right\}, \\
& C_{13}^{\text {Sat }}=\frac{\lambda}{D}\left\{d_{1} \theta+\frac{K_{F}}{\varphi K_{g} \lambda}\left[\lambda_{1} \alpha^{\prime}-\frac{8 \mu^{2} \alpha_{0} \Delta_{N}}{9 L}\right]\right\}, \\
& C_{44}^{\text {Sat }}=\mu, \\
& \text { and, } C_{55}^{\text {Sat }}=\mu\left(1-\Delta_{T}\right) .
\end{aligned}
$$

Accurate expression for the parameters $L, L_{1}, D, d_{1}, d_{2}, \alpha_{0}$, $\alpha, \lambda_{1}$ etc. used in equations 5-9 can be found in Brown and 


\section{Fluid substitution in fractured medium}

Gurevich (2004). Using equations 5-9 we perform our fluid substitution analysis.

\section{Example}

For the purpose of investigation of the effect of fluid substitution in a porous HTI medium, we consider a twolayer case. In our model, the top layer comprises isotropic hard shale with $\mathrm{P}$-wave velocity $=3.85 \mathrm{~km} / \mathrm{s}$, S-wave velocity $=1.45 \mathrm{~km} / \mathrm{s}$, and mineral density $=2.70 \mathrm{gm} / \mathrm{cc}$. Our bottom layer is a fractured class 3 sand (HTI medium) with dry $\mathrm{P}$-wave velocity $=3.00 \mathrm{~km} / \mathrm{s}$, dry $\mathrm{S}$-wave velocity $=1.75$ $\mathrm{km} / \mathrm{s}$, mineral density $=2.65 \mathrm{gm} / \mathrm{cc}$; the fracture density is 0.08 for case 1 and 0.16 for case 2, and the porosity is $20 \%$. We have not considered any porosity (or fluid substitution effect) in the top shale layer. With the above parameters, we calculate the stiffness matrix elements of the dry HTI medium by linear slip theory (Schoenberg and Sayers, 1995) where dry velocities and density are used to calculate background isotropic properties. Then, using equations 5 to 9 , saturated stiffness elements are calculated for our analysis.

Figure 1a shows the variation of $\mathrm{P}$-wave moduli $\left(\mathrm{C}_{11}\right.$ and $\mathrm{C}_{33}$ ) with porosity for the case 1 (fracture density 0.08 ). Figure 1b shows the same plot for the case 2 (fracture density is doubled to 0.16 ). In both cases we observe a decrease (almost 40\%) in the P-wave moduli with increasing porosity. This phenomenon is similar to the isotropic case. Increase in the fracture density (Figure 1b) overall causes a decrease in the value of the moduli. This effect is more prominent on $\mathrm{C}_{11}$ than $\mathrm{C}_{33}$.

In Figure 1c we show the effect of porosity on vertical and horizontal P-wave velocities for lower fracture density (case 1), and Figure 1d shows a similar plot for the higher fracture density medium (case 2 ). In both the cases, P-wave velocities tend to decrease with increasing porosity. We observe a slight increment in the velocities for the higher porosity values $(25 \%$ to $35 \%)$. This is due to the steep density gradient from the porosity-density relationship used in this model. Similar to the observation in Figures 1a, and $\mathrm{b}$, here also we note that increasing fracture density lowers the P-wave velocities. The effect is more dominant on the horizontal velocity than on the vertical velocity. In the next plot (Figure 1e) we show the variation of three anisotropic parameters $\left(\varepsilon^{v}, \gamma^{v}\right.$ and $\left.\delta^{v}\right)$ for the HTI medium (as defined by Rüger, 1997) as a function of porosity for the case 1. As expected from the theory, there is no change observed in $\gamma^{v}$ with porosity. There is a slight decrease in $\varepsilon^{v}$ (but increase in magnitude) and considerable increase in $\delta^{v}$ (but decrease in magnitude). In case 2 when fracture density is high, as expected, we observe an overall increase in magnitude of all the anisotropic parameters (Figure 1f). However, their variation with porosity remains the same.
In Figure 2, we investigate the relation between porosity and reflection coefficients. The left panel of Figure 2a shows a plot of reflections coefficients as functions of incident angle (x-axis) and azimuth (y-axis) for case 1. In this case the sand has $5 \%$ porosity and is $100 \%$ water saturated. Sinusoidal variation of reflection coefficients (Mallick et al., 1998) due to anisotropy is visible at large incident angles (greater than $30^{\circ}$ ). In the right panel, we show reflection coefficient as a function of incident angle for two azimuths $0^{\circ}$ (symmetry plane) and $90^{\circ}$ (isotropy plane). Figure $2 \mathrm{~b}$ shows similar plot for $35 \%$ porosity and case 1 . Comparing figures $2 \mathrm{a}$ and $2 \mathrm{~b}$ we notice that the value of reflection coefficient decreases and its magnitude increases with increased porosity. Also, the left panel of Figure $2 b$ shows that the variation due to anisotropy becomes more visible at lower incident angle for higher porosity case. In figure $2 \mathrm{c}$ we show the lower porosity (porosity 5\%) reflection coefficient for higher fracture density (Case 2, fracture density 0.16 ). We can observe that increase in fracture density overall lowers the value of reflection coefficients (hence increases its magnitude). Figure $2 \mathrm{~d}$ shows the higher porosity (porosity 35\%) reflection coefficients as function of azimuth and incident angle. Due to increase in porosity, the reflection coefficients decreases in value and its magnitude increases. This observation is consistent with the velocity-porosity relation we observe in figure $1 \mathrm{~d}$.

We further investigate the influence of water saturation and crack density on P-wave moduli, P-wave velocity, anisotropy parameters, and reflection coefficients. We observe that increase in water saturations increases the value of P-wave moduli and velocities. Hence reflection coefficients also increase with water saturation. Effect of azimuthal anisotropy becomes more visible in water saturated case than the dry case. Different anisotropic parameters behave differently with changes in water saturation. Results from water saturation analysis are not included in the current paper.

\section{Conclusions}

We performed limited sensitivity analysis of Gurevich's (2003) porous HTI medium fluid substitution equations for a class 3 sand. We observe that just like isotropic cases, Pwave moduli, and P-wave velocities decreases with increasing porosity in anisotropic HTI medium. Increasing crack density lowers these values further. The effect is more pronounced on horizontal component than the vertical component. The anisotropic parameters also change with porosity. Since we observe a decrease in velocity with porosity, we also observe a decrease in the value of reflection coefficients with porosity. The effect of azimuthal anisotropy becomes more prominent for the higher porosity cases than for the lower porosity. 


\section{Fluid substitution in fractured medium}
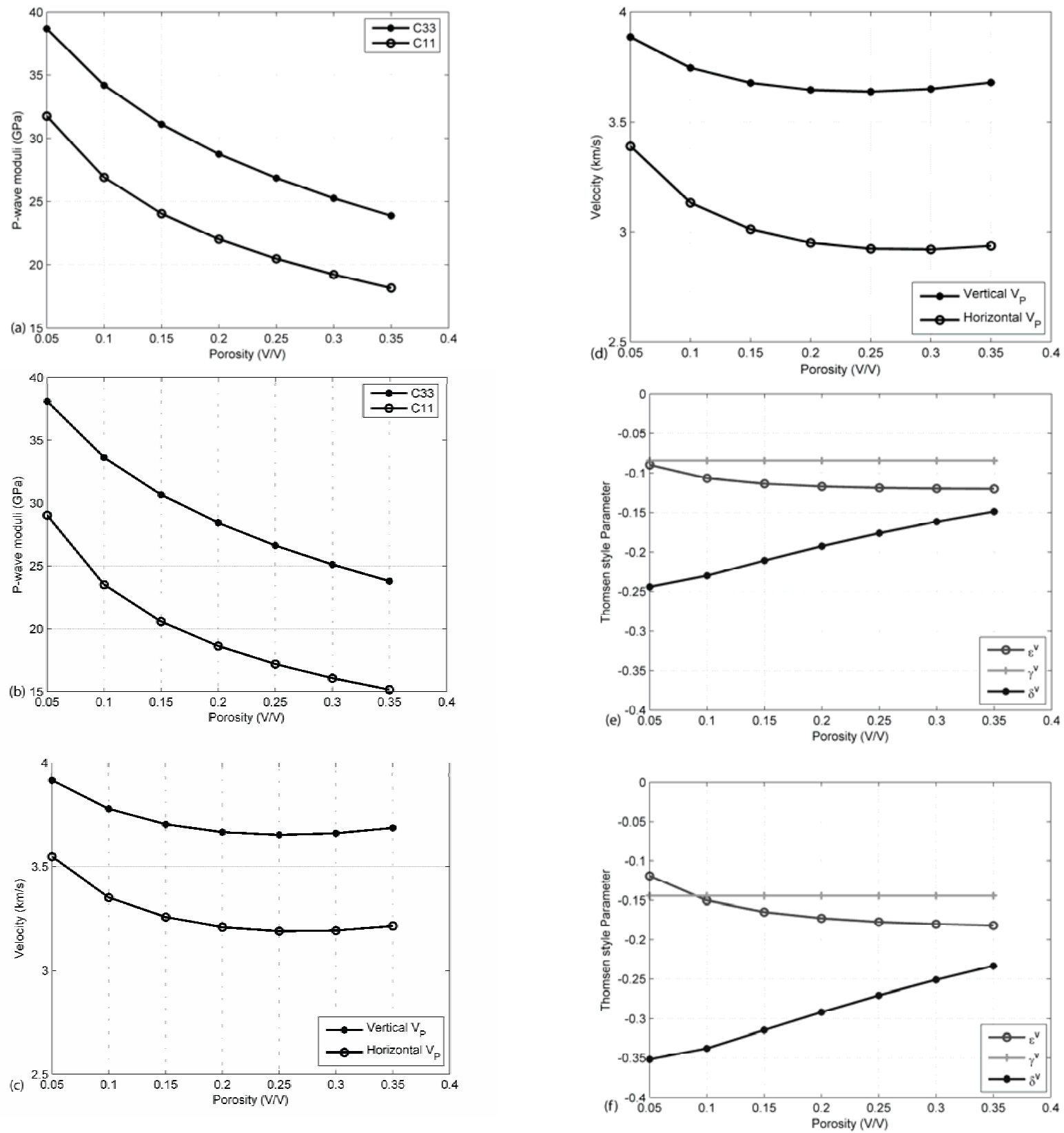

Figure 1: (a) variation of P-wave moduli with porosity for the fracture density 0.08 (case 1), (b) similar result for higher crack density (0.16, case 2). (c) and (d) plots of velocity variation as a function of porosity for case 1 and 2 respectively. (e) and (f) are the variation of anisotropic parameters as function of porosity for case 1 and 2 respectively. Please see the text for details. 


\section{Fluid substitution in fractured medium}
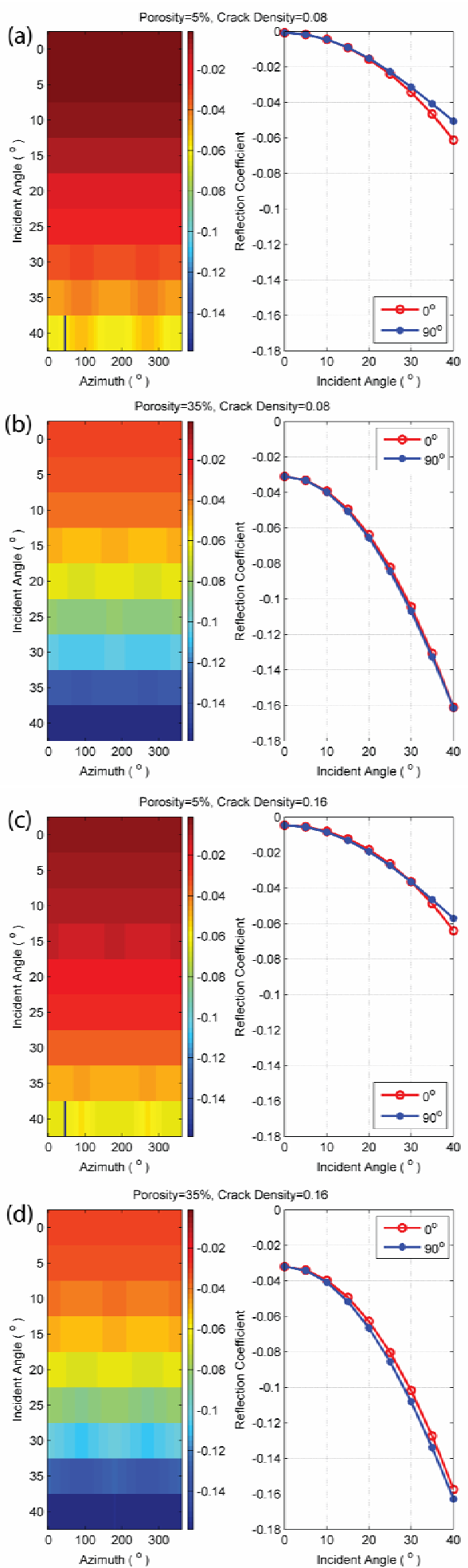

Figure 2: Left panel of Plot (a) shows the variation of reflection coefficients as function of incident angle (y-axis) and azimuth ( $\mathrm{x}$ axis). Right panel shows the variation of reflection coefficients with incident angle for $90^{\circ}$ azimuth (isotropy plane) and $0^{\circ}$ azimuth (symmetry plane). This plot is for $5 \%$ porosity and lower fracture density $(0.08$, case 1$)$. Plot (b) is similar plot for the $35 \%$ porosity and lower fracture density. Plot (c) is again the same plot with $5 \%$ porosity but higher fracture density $(0.16$, case 2$)$. Last plot (d) shows the similar figure for $35 \%$ porosity and higher crack density. Detailed analysis of these plots are described in the text.

\section{Acknowledgments}

We are thankful to ConocoPhillips for allowing us to present our work at SEG. Help from Utpalendu Kuila during his summer internship at ConocoPhillips and other members of the seismic technology group are also appreciated. 


\section{EDITED REFERENCES}

Note: This reference list is a copy-edited version of the reference list submitted by the author. Reference lists for the 2010 SEG Technical Program Expanded Abstracts have been copy edited so that references provided with the online metadata for each paper will achieve a high degree of linking to cited sources that appear on the Web.

\section{REFERENCES}

Brown, L., and B. Gurevich, 2004, Frequency dependent seismic anisotropy of porous rocks with pennyshaped cracks: Exploration Geophysics, 35, no. 2, 111-115, doi:10.1071/EG04111.

Brown, R., and J. Korringa, 1975, On the dependence of the elastic properties of a porous rock on the compressibility of the pore fluid: Geophysics, 40, 608-616, doi:10.1190/1.1440551.

Gassmann, F., 1951, Uber die Elastizitat poroser Medien: Vierteljahrsschrift der Naturforschende Gesellschaft, 96, 1-3 (in German; English translation by Berryman, J., J. Castagna, and C. Ecker, 2007, On elasticity of porous media: Classics of Elastic Wave Theory, SEG, 389-07.)

Gurevich, B., 2003, Elastic properties of saturated porous rocks with aligned fractures: Journal of Applied Geophysics, 54, no. 3-4, 203-218, doi:10.1016/j.jappgeo.2002.11.002.

Mallick, S., K. Craft, L. Meister, and R. E. Chambers, 1998, Determination of the principal directions of azimuthal anisotropy from P-wave seismic data: Geophysics, 63, 692-706, doi:10.1190/1.1444369.

Nelson,R.A.,2001, Geologic Analysis of Naturally Fractured Reservoirs: Gulf Professional Publishing.

Rüger, A., 1997, P-wave reflection coefficients for transversely isotropic media with vertical and horizontal axis of symmetry: Geophysics, 62, 713-722, doi:10.1190/1.1444181.

Schoenberg, M. A., and C. M. Sayers, 1995, Seismic anisotropy of fractured rock: Geophysics, 60, 204211, doi:10.1190/1.1443748. 